\title{
Relativistic Newtonian Gravitation That Gives the Correct Prediction of Mercury Precession
}

\author{
Espen Gaarder Haug \\ Norwegian University of Life Sciences, Ås, Norway \\ Email: espenhaug@mac.com
}

How to cite this paper: Haug, E.G. (2020) Relativistic Newtonian Gravitation That Gives the Correct Prediction of Mercury Precession. Journal of High Energy Physics, Gravitation and Cosmology, 6, 238-243. https://doi.org/10.4236/jhepgc.2020.62017

Received: March 5, 2020

Accepted: March 29, 2020

Published: April 2, 2020

Copyright (อ 2020 by author(s) and Scientific Research Publishing Inc. This work is licensed under the Creative Commons Attribution International License (CC BY 4.0).

http://creativecommons.org/licenses/by/4.0/

\begin{abstract}
In the past, there was an attempt to modify Newton's gravitational theory, in a simple way, to consider relativistic effects. The approach was "abandoned" mainly because it predicted only half of Mercury's precession. Here we will revisit this method and see how a small logical extension can lead to a relativistic Newtonian theory that predicts the perihelion precession of Mercury correctly.
\end{abstract}

\section{Keywords}

Newton Gravity, Relativistic Adjustment, Precession of Mercury

\section{Introduction}

In 1981 and 1986, Bagge [1] and Phillips [2] each suggested an ad-hoc modification of Newton by simply replacing the smaller mass in the formula with a relativistic mass

$$
F=G \frac{M \frac{m}{\sqrt{1-\frac{v^{2}}{c^{2}}}}}{r^{2}}
$$

The velocity $v$ is the relative velocity between the two gravitational objects: the velocity of Mercury relative to the Sun, for example. Phillips initially claimed that his derivation, based on this, led to a prediction of the perihelion precession of Mercury equal to that of Einstein's general relativity theory [3]. However, according to criticism from Ghosal in 1987, this approach leads to a perihelion precession of Mercury that is too low. The method has also been criticized by Chow [4] for the same reason. Peters [5] claims that Philipps made a mistake in his Mercury perihelion derivation and that, in reality, his prediction only gives 
half of the prediction as GR (the GR prediction has been observed). Philipps openly admitted this and discussed his mistakes in detail [6]. He was clear that his theory underestimated the perihelion precession of Mercury, but noted that further adjustments to the theory could potentially be done in the future. Biswas [7] published an interesting paper titled "Special Relativistic Newtonian Gravity" where he claimed:

The resulting theory is significantly different from the general theory of relativity. However, all known experimental results (precession of planetary orbits, bending of the path of light near the Sun, and gravitational spectral shift) are still explained by this theory.

However, Peters [8] then pointed out that Biswas had also made a mistake in his derivation, something Biswas agreed to in correspondence with Peters. Ghosal and Cakraborty [9] agree on the criticism of Biswas, but claim his idea was still interesting. Here we will follow up on this discussion and show that there is a simple and logical way to extend this approach in a fruitful manner.

\section{Modified Relativistic Newtonian Gravity That Gives the Correct Prediction of the Precession of Mercury}

In the relativistic extension of Newton given by [1] [2],

$$
F=G \frac{M \frac{m}{\sqrt{1-\frac{v^{2}}{c^{2}}}}}{r^{2}}
$$

The velocity $v$ must be interpreted as the velocity between the large and small masses. This extension is, in our view, only valid when the gravity phenomenon is observed from the frame of the large gravitational object, such as predicting the orbital velocity of the moon relative to the Earth, for example. In this case under consideration, however, the small relativistic mass will fall out and we get the same predictions as in standard Newtonian gravity. When it comes to gravity phenomena between two masses as observed from a third frame, we claim it is logical to complete additional ad hoc modifications to the formula above. When observing the Sun's gravitational influence on Mercury, for example, we must also consider the Sun's velocity relative to us as we observe it from Earth. We suggest the following modification

$$
F=G \frac{\frac{m}{\sqrt{1-\frac{v_{m}^{2}}{c^{2}}}} \frac{M}{\sqrt{1-\frac{v_{M}^{2}}{c^{2}}}}}{r^{2}\left(1-\frac{v_{M}^{2}}{c^{2}}\right)}
$$

where $v_{m}$ and $v_{M}$ are the velocities of the large and small masses as observed from the observer frame, that is to say, in our case, from Earth. As can be seen in our formula, we are suggesting that $r$ (center to center between the two gravitational masses) should be the length contracted depending on the velocity of the 
two objects relative to the observer; this is best approximated by the velocity of the large gravitational object relative to the observer frame. For example, assume a galaxy with distance $r$ between the galactic center and one of the stars in the arm of the galaxy, as observed from the galactic center. We claim that this distance likely will appear to be contracted, as observed from Earth and as measured with Einstein-Poincaré synchronized clocks. Its contracted length will follow standard Lorentz length contraction, in our formulation, and will be $r \sqrt{1-\frac{v_{M}^{2}}{c^{2}}}$. That is to say, for fast-moving galaxies we have two effects that lead to stronger gravity than predicted by the Newtonian theory. The first effect is that the relativistic mass is relevant for gravity (and this mass is larger than the rest-mass), and the second effect is that the distance center to center between the gravity objects must appear to be contracted, as observed from the laboratory (typically the Earth).

In 1859, LeVerrier pointed out that the perihelion of Mercury evidently precesses at a slightly faster rate than predicted by Newtonian mechanics. The Lagrangian is given by

$$
L=T-V
$$

This gives

$$
L=\frac{m c^{2}}{\sqrt{1-\frac{v_{m}^{2}}{c^{2}}}}+G \frac{M \frac{m}{\sqrt{1-\frac{v_{m}^{2}}{c^{2}}}}}{r\left(1-\frac{v_{M}^{2}}{c^{2}}\right)}
$$

when $v_{M} \square c$, we can use a Taylor series expansion and get

$$
\begin{gathered}
L=\frac{m c^{2}}{\sqrt{1-\frac{v_{m}^{2}}{c^{2}}}}+G \frac{M \frac{m}{\sqrt{1-\frac{v_{m}^{2}}{c^{2}}}}}{r}+\frac{v_{M}^{2}}{c^{2}} G \frac{M \frac{m}{\sqrt{1-\frac{v_{m}^{2}}{c^{2}}}}}{r} \\
L=\frac{m c^{2}}{\sqrt{1-\frac{v_{m}^{2}}{c^{2}}}}+\frac{G M m}{r \sqrt{1-\frac{v_{m}^{2}}{c^{2}}}}+\frac{v_{M}^{2}}{c^{2}} \frac{G M m}{r \sqrt{1-\frac{v_{m}^{2}}{c^{2}}}}
\end{gathered}
$$

And to simplify further, we can set $k=G M m$ and this gives

$$
L=\frac{m c^{2}}{\sqrt{1-\frac{v_{m}^{2}}{c^{2}}}}+\frac{k}{r \sqrt{1-\frac{v_{m}^{2}}{c^{2}}}}+\frac{v_{M}^{2}}{c^{2}} \frac{k}{r \sqrt{1-\frac{v_{m}^{2}}{c^{2}}}}
$$

Next assume that $v_{m} \square c$ and $v_{m} \approx v_{M}$, we can then use a Taylor series expansion and we get

$$
L=m c^{2}+\frac{1}{2} m v_{M}^{2}+\frac{k}{r}+\frac{3}{2} \frac{v_{M}^{2}}{c^{2}} \frac{k}{r}+O\left(c^{-4}\right)
$$

given extensive calculations, this seems to lead to the same prediction as GR for Mercury precession, that is 


$$
\delta=\frac{6 \pi m}{c^{2} a\left(1-e^{2}\right)}
$$

\section{Equivalence Principle}

Einstein's [10] equivalence principle basically states that inertial mass and gravitational mass are the same thing. It is considered a well-tested concept, at least inside a wide range of observational values. In the case of two reference frames, we have

$$
\begin{gathered}
F=\frac{m}{\sqrt{1-\frac{v_{m}^{2}}{c^{2}}}} a=G \frac{M \frac{m}{\sqrt{1-\frac{v_{m}^{2}}{c^{2}}}}}{r^{2}} \\
F=m a=G \frac{M m}{r^{2}}
\end{gathered}
$$

which means we have

$$
a=G \frac{M}{r^{2}}
$$

So, we see it gives exactly the same result as standard theory in this case. A discussion of whether or not this is only valid for a weak gravitational field is outside the scope of this paper. All direct measurements of the equivalence principle have, to our knowledge, been done in a two frame observational setting, so our theory predicts the same here as standard theory. In the case of three reference frames, we have

$$
F=\frac{m}{\sqrt{1-\frac{v_{m}^{2}}{c^{2}}}} a=G \frac{M \frac{m}{\sqrt{1-\frac{v_{m}^{2}}{c^{2}}}}}{r^{2}\left(1-\frac{v_{M}^{2}}{c^{2}}\right)^{3 / 2}}
$$

which means we have

$$
a=G \frac{M}{r^{2}\left(1-\frac{v_{M}^{2}}{c^{2}}\right)^{3 / 2}}
$$

This will indeed give a different predicted acceleration of an object $m$ relative to object $M$, as observed from a third frame. Such experiments have not been done directly to our knowledge, but possibly indirectly through cosmological observations. Therefore, this should be of interest for possible alternative interpretations of cosmological observations. This would also be significantly different from standard theory when $v_{M}$ has a significant velocity relative to Earth. Intuitively, this should mean the red-shift may be likely higher than expected when excluding the hypothesis of expanding space. All in all, we think our 
theory therefore should be highly relevant for further studies. Our model also indicates that galaxy arms should rotate somewhat faster than predicted from standard theory by taking only baryonic matter into account. However, we do not claim that this is enough alone to account for the missing dark matter. This is however a discussion outside the scope of this article. The focus here is that Newton relativistic modifications may have been rejected too early in relation to predictions of Mercury's precession. Still, we think our theory does not conflict with observations that have been completed in relation to the equivalence principle. Naturally, we are open to further discussions on this. After all, physics can only progress by exploring, testing, and scrutinizing any new ideas carefully.

\section{Summary}

In the past, several ad hoc modifications of Newton's gravity theory have been proposed and discussed. These approaches have been criticized for predicting only half of the perihelion of the precession of Mercury. Taking that work as a start, however, we have suggested some logical extensions to this theory. If we are looking at relativistic effects, they should be evaluated from the observer frame. In this case, when the gravity phenomenon is not observed from the large gravity mass itself, but rather from an outside frame such as the Earth, then we also must take into account the velocity of the Sun relative to the Earth. After completing an ad hoc adjustment accordingly, we find the same prediction of precession of Mercury as general relativity theory predicts. Although we have not tested these results further, we think this is interesting enough to require further investigation, and hope this paper will highlight the way for future research.

\section{Conflicts of Interest}

The author declares no conflicts of interest regarding the publication of this paper.

\section{References}

[1] Bagge, E.R. (1981) Relativistic Effects in the Solar System. Atomkernenergie Kerntechnik, 39, 223-228.

[2] Phipps, T.E. (1986) Mercury's Precession According to Special Relativity. American Journal of Physics, 54, 245-247. https://doi.org/10.1119/1.14664

[3] Einstein, A. (1916) Näherungsweise integration der feldgleichungen der gravitation. Sitzungsberichte der Königlich Preussischen Akademie der Wissenschaften Berlin.

[4] Chow, T. (1992) On Relativistic Newtonian Gravity. European Journal of Physics, 13, 198. https://doi.org/10.1088/0143-0807/13/4/010

[5] Peters, P.C. (1986) Comment on “Mercury's Precession According to Special Relativity”. American Journal of Physics, 55, 757. https://doi.org/10.1119/1.15014

[6] Phipps, T.E. (1986) Response to “Comment on 'Mercury's Precession According to Special Relativity”. American Journal of Physics, 55, 758-759. https://doi.org/10.1119/1.15015 
[7] Biswas, T. (1988) Minimally Relativistic Newtonian Gravity. American Journal of Physics, 56, 1032. https://doi.org/10.1119/1.15385

[8] Peters, P.C. (1990) Comment on "Minimally Relativistic Newtonian Gravity”. American Journal of Physics, 56, 188. https://doi.org/10.1119/1.16183

[9] Ghosal, S.K. and Chakraborty, P. (1991) Relativistic Newtonian Gravity: An Improved Version. European Journal of Physics, 12, No. 6. https://doi.org/10.1088/0143-0807/12/6/003

[10] Einstein, A. (1922) The Meaning of Relativity. Princeton University Press, Princeton, NJ. 